\title{
"EN TEORÍA, ES PERFECTO”. LA PARTÍCULA EN TEORIA: DEBILITAMIENTO ASERTIVO, MARCACIÓN ARGUMENTATIVA DE TIPO PREDICTIVO Y PATRONES DISCURSIVOS*
}

\author{
"IN THEORY, IT IS PERFECT". THE PARTICLE IN THEORY: \\ ASSERTIVE WEAKENING, PREDICTIVE ARGUMENTATIVE \\ MARKERS, AND DISCOURSIVE PATTERNS
}

\section{ESTRELLA MONTOLÍO DURÁN \\ Lengua Española. Departamento de Filología Hispánica. \\ Universidad de Barcelona.emontoliod@ub.edu}

\section{RESUMEN}

La locución adverbial en teoría funciona en determinados contextos sintácticos como una partícula discursiva de carácter modal epistémico de debilitamiento asertivo. En contextos más específicos puede actuar también como un operador argumentativo, de acuerdo con la definición que la Teoría de la Argumentación prevé para estos elementos. En concreto, sostenemos que en teoría, al igual que en principio y otras partículas semejantes de origen temporal, funciona en alguno de sus usos como un operador de debilitamiento argumentativo. El valor pragmático de debilitamiento asertivo que aporta esta partícula puede explicarse a partir del significado relacional del sustantivo teoría, que incluye 'teoría versus práctica', a partir del cual la partícula discursiva en teoría convoca siempre una alternativa, otro punto de vista. En particular, el fragmento que señala este marcador se presenta como contrastado con otra alternativa posible, la correspondiente a la esfera de la práctica o realidad. Esa esfera alternativa, en ocasiones, es convocada de manera inferencial; pero en otros casos aparece de manera explícita en la prosecución del discurso, dando pie a un patrón discursivo recurrente, tanto en registros orales como escritos: [en teoría A. Pero (en la práctica) B]. De ahí que la partícula en teoría pueda considerase como un marcador de contraargumentación de tipo predictivo (anticipativo).

Palabras clave: Partícula discursiva, marcador del discurso, operador argumentativo, pragmática, argumentación, polifonía, marcación predictiva, patrón discursivo.

* Este trabajo se enmarca en el proyecto Estrategias de textualidad del discurso profesional en soportes multimodales. Análisis y propuestas de mejora (Referencia: FFI2012-28933), financiado por el Ministerio de Economía y Competitividad así como por los fondos FEDER. 


\begin{abstract}
In certain syntactic contexts, the adverbial expression in theory operates works as a modal discourse particle that weakens the assertion. It can also work as an argumentative operator, according to the Argumentation Theory's definition. Specifically, we argue that, as in principle and similar discourse particles with temporal origin, in theory may work in one of its uses as an argumentative weakening operator. The pragmatic value provided by this particle can be explained taking into account the relational meaning of the noun theory, which includes 'theory versus practice'. Considering this lexical meaning, the discourse particle in theory always calls up an alternative; i.e., another point of view. In particular, the fragment pointed by the discourse marker appears as contrasted with another alternative that refers to the practice or reality. This alternative can be either conveyed through an inference or be explicitly expressed. The latter gives rise to a recurrent discourse pattern, present both in oral and in written corpora: [in theory, A. But (in the practice) B]. Hence, the particle in theory can considered as a predictive counterargumentative marker.
\end{abstract}

Keywords: Discourse particle, discourse marker, argumentative operator, pragmatics, argumentation, polyphony, predictive structures, discourse pattern.

Recibido: 23.03.2016. Aceptado: 02.05.2016.

\title{
1. INTRODUCCIÓN ${ }^{1}$
}

La producción bibliográfica generada en el ámbito hispánico sobre los marcadores del discurso ha sido ciertamente importante a lo largo de las últimas dos décadas. Estos trabajos se han ocupado, por lo general, de las partículas que implican una relación de contigüidad entre la instrucción planteada por el marcador y los miembros del discurso a los que alcanza. Resulta evidente que, en algunos casos, tal relación de adyacencia entre partícula y miembros del discurso viene obligada por las características gramaticales de la partícula; así ocurre con las conjunciones. De este modo, por ejemplo, un conector conjuntivo como asi que codifica una instrucción de primer orden de señalización de 'consecuencia' que afecta al miembro adyacente que le sigue, $y$, complementariamente, una instrucción derivada o de segundo orden de marca de 'causa' al miembro precedente':

\footnotetext{
${ }^{1}$ Agradezco a mis colegas José Portolés, Pedro Gras, Anna López y Fernando Polanco su lectura atenta de este trabajo y sus atinadas observaciones. Las inexactitudes que pueda contener son de mi estricta responsabilidad.

${ }^{2}$ Sobre el valor fórico de los marcadores del discurso, pueden consultarse, entre otros Cuenca (1990), Fuentes (1987), Martín Zorraquino y Portolés (1999), Portolés (1999) y (2004).
} 
(1) Pepe lleva tres días acostándose a las tantas, así que no creo que quiera salir esta noche con nosotros. [enunciado señalado retroactivamente como 'causa'] [conector] [enunciado marcado como 'consecuencia']

Diferente es el caso, sin embargo, de los marcadores "parentéticos" (Cuenca, 2001; Montolío, 2001) cuya autonomía sintáctica les permite movilidad en el interior del enunciado. En realidad, sin embargo, la mayor parte de los marcadores parentéticos suelen situarse al inicio del segundo miembro, o, incluso, restringen su ubicación a esta sola posición -es el caso de, por ejemplo, ahora bien (Martín Zorraquino y Portolés, 1999)-. Se observa, pues, una preferencia por la posición al inicio del segundo miembro, siguiendo el esquema [A conector B], en el que el conector mantiene una relación de contigüidad con los fragmentos del discurso a los que señala. Este esquema de marcación ha sido, sin duda, el que ha concentrado la atención de los especialistas en sus trabajos, probablemente porque constituye un esquema prototípico de conexión entre miembros del discurso, sean éstos cláusulas, oraciones o secuencias de oraciones.

Ahora bien, el análisis de los discursos tanto orales como escritos muestra que existen también casos de marcación argumentativa de tipo predictivo o anticipativo, sin inmediación sintáctica necesaria. En este sentido, un grupo de marcadores que presentan interés para la investigación por el tipo de cohesión discursiva peculiar que realizan entre miembros no necesariamente contiguos son los operadores de debilitamiento argumentativo ${ }^{3}$ (Montolío, 2003, 2011, 2014).

El objetivo de esta contribución es doble: por un lado, abrir una vía de investigación sobre la marcación predictiva (esto es, catafórica y no necesariamente en relación de contigüidad) de las relaciones argumentativas, discursivas o interactivas que se producen en todo tipo de registros, a semejanza de la investigación sobre mecanismos anticipativos que algunos especialistas han planteado para los textos escritos planificados (Tadros, 1994; Hyland, 2005) -y que sólo recientemente se está apuntando para el español (Hyde, 1999; Montolío, 2016; Yúfera, 2014, entre otros)-.

Por otro lado, en el presente trabajo se propone profundizar en el análisis de la marcación anticipativa de contraargumentación que, con frecuencia, realiza la clase de marcadores de los operadores de debilitamiento, partículas para las que se ha propuesto esta función predictiva en trabajos previos. En particular, el objetivo es ahondar en la descripción de la unidad en teoría. Sobre este marcador se propone analizar: (i) su forma; (ii) su significado y el esquema de interpretación que acarrea; (iii) su valor argumentativo en tanto que operador de debilitamiento; (iv)

\footnotetext{
${ }^{3}$ Los trabajos especializados sobre marcadores del discurso han identificado tres tipos de operadores argumentativos para el español: (i) de concreción (por ejemplo, en particular y similares), (ii) de refuerzo argumentativo (en realidad, en el fondo, de hecho) (Portolés 1998: 9.2.4; Martín Zorraquino y Portolés 1999: 63.5) y (iii) de formulación (bueno) (Portolés 2004: 291); (vamos, vaya) (Polanco 2013, 2014), a los que cabe añadir (iv) de debilitamiento argumentativo (en principio, de momento, por el momento, por ahora, en teoría) (Montolío 2003, 2011 y 2014).
} 
su función como marca predictiva de contraargumentación; y (v) los esquemas o patrones discursivos en los que prototípicamente se inserta.

\section{CORPUS}

Los ejemplos utilizados en esta investigación proceden de tres corpus. De un lado, el corpus de conversaciones coloquiales grabadas y transcritas por Briz y el equipo Val.Es.Co (2002). De otro, se ha manejado también el corpus PRESEEA correspondiente a los hablantes de la ciudad de Alcalá (Moreno, Cestero, Molina y Paredes, 2002). Asimismo, se ha utilizado también el Corpus de Referencia del Español Actual (CREA) de la Real Academia Española de la Lengua, empleando las siguientes variables básicas: país, España; años, 1995-2011; tema, todos; medio, Oral.

A fin de disponer de más ejemplos, se han seleccionado también los procedentes del medio "Miscelánea”, ya que contiene numerosos fragmentos de textos web, cuyo registro presenta numerosos mecanismos lingüísticos de coloquialidad. Asimismo, con el objetivo de comprobar que no se trata de un mecanismo propio estrictamente de la variedad de español peninsular, se han manejado igualmente ejemplos procedentes de Chile. Finalmente, se han aportado también ejemplos de lengua escrita que permiten confirmar el uso transversal, en todo tipo de registros, del marcador en teoría, al tiempo que corroborar la funcionalidad de los mecanismos textuales de carácter anticipativo en la elaboración de textos planificados.

Globalmente, se han analizado 80 ocurrencias de esta partícula, que se reparten del siguiente modo: corpus Val.Es.Co, tres ocurrencias; corpus PRESEEA, hablantes de instrucción superior, un caso; de instrucción media, tres casos y de instrucción primaria, cero. En cuanto al corpus CREA, en la selección de Chile, proporciona 19 ocurrencias. En la opción España, aporta ocho casos para medio Oral y ocho para medio Miscelánea. Respecto al medio escrito (libros y periódicos), ofrece 517 casos, de los que hemos analizado 40.

\section{SOBRE LA UNIDAD EN TEORÍA}

$\mathrm{Al}$ igual que otros sintagmas preposicionales que desarrollan valores discursivos, la unidad en teoría presenta un indudable interés para el estudio del léxico, la gramática y la pragmática. En algunos contextos, en teoría puede analizarse como una locución adverbial (así aparece descrita en obras lexicográficas como el DRAE, 1992; Seco, Andrés y Ramos, 1999; Martínez y Myre, 2009) de carácter modal ("pararrespectual" añade Santos Río, 2003). Fuentes, en su trabajo sobre conectores y operadores del español (2009), la considera un operador modal. En este 
trabajo se sostendrá que, en algunos contextos, funciona como operador argumentativo de debilitamiento.

En este sentido, dos ejemplos que aporta el Diccionario del español actual de Seco et al. (1999) para esta expresión permiten ilustrar dos de sus funciones claramente distintas; la de complemento verbal (ejemplo (2)) y la de partícula dis$\operatorname{cursiva}^{4}(3)$ :

(2) Ya organizadas las situaciones en teoría, intentamos recrear la estructura de una sesión psicodramática.

(3) En teoría, íbamos a reunirnos a las cinco.

Como se verá en los apartados que siguen, en buena parte de sus usos como partícula, en teoría funciona, de hecho, como un operador argumentativo de debilitamiento.

La noción de operador argumentativo, matizada por el propio Ducrot tras el planteamiento general de la Teoría de la Argumentación (Anscombre y Ducrot, 1983), atiende a los marcadores del discurso cuyas instrucciones semánticopragmáticas no sirven para conectar enunciados entre sí, sino cuyo alcance es un único enunciado. Los operadores determinan la orientación argumentativa de los enunciados en los que intervienen, de tal modo que la intención argumentativa y, por tanto, la prosecución natural del discurso ya no puede ser la misma para el enunciado una vez ha sido incluido en su interior un operador argumentativo. En cómoda consonancia con esta definición, operadores de debilitamiento ya estudiados en trabajos anteriores, como en principio, condicionan también las posibilidades discursivas del enunciado en el que se insertan.

En este trabajo se defenderá que, al igual que en principio y partículas semejantes de origen temporal, en teoría funciona también como un operador de debilitamiento, como puede comprobarse al contrastar la diferente adecuación de las siguientes versiones (a) y (b):

(4a) La doctora Giménez es una buena dermatóloga. Estoy plenamente convencida de que solucionará tus problemas de piel.

(4b) \#La doctora Giménez es una buena dermatóloga, en teoría. Estoy plenamente convencida de que solucionará tus problemas de piel.

Como muestran las dos versiones de (4), cuando se inserta el operador en teoría (4b), se debilita la fuerza asertiva del enunciado. De hecho, la inserción de en

\footnotetext{
${ }^{4}$ Se usa aquí la denominación partícula discursiva en el mismo sentido que en la obra colectiva coordinada por Briz, Pons y Portolés (2008) para referirse a cualquier palabra invariable o locución cuyo significado sea procedimental y sirva para guiar las inferencias de las palabras con significado conceptual.
} 
teoría, además de debilitar la aserción, permite en ocasiones que la orientación argumentativa del enunciado pueda incluso variar y desviarse del topos propio de la proposición a la que acompaña la partícula, en este caso, "ser una buena médica", cuya conclusión lógica es "curar las dolencias de sus pacientes", conclusión que queda debilitada con la inserción del operador, como muestra la extrañeza de (4b).

Como se ve, en teoría permite proseguir el discurso con un segundo miembro antiorientado respecto del primero. Es decir, a veces en teoría invita a la inversión argumentativa, como refleja la adecuación de la versión siguiente (4d).

(4c) \#La doctora Giménez es una buena dermatóloga. Pero ya veremos si es capaz de ayudarte con tus problemas de piel.

(4d) La doctora Giménez es una buena dermatóloga, en teoría. Pero ya veremos si es capaz de ayudarte con tus problemas de piel.

La versión (4c) del ejemplo (4) demuestra que el enunciado deviene incoherente si aparece un segundo miembro argumentativamente antiorientado respecto del primero cuando no hay ningún tópico que explique dicha contraposición, ni tampoco marca argumentativa alguna que la anuncie. En cambio, en la correspondiente versión (4d), en la que se ha insertado el operador en teoría, la antiorientación del segundo miembro respecto del previo resulta aceptable precisamente porque el operador ha indicado la debilidad argumentativa del enunciado al que acompaña y, por tanto, ha favorecido la prosecución del discurso en términos de antiorientación respecto del segmento discursivo previo.

En publicaciones anteriores se ha descrito con exhaustividad las características gramaticales y pragmáticas de un conjunto de partículas discursivas (por un lado, en principio; y, por otro, por el momento, por ahora, de momento) que se han agrupado bajo la denominación operadores argumentativos de debilitamiento. Como muestran los ejemplos que se manejan a lo largo de este trabajo, al igual que estos operadores de debilitamiento, el marcador en teoría puede aparecer en todo tipo de oraciones (independientes, coordinadas, subordinadas); tanto en oraciones con verbo en indicativo, como con verbo en subjuntivo.

Por otro lado, en teoría presenta gran libertad posicional: puede aparecer en los márgenes del enunciado, tanto en posición inicial (ejemplos (10) y (17)) como en posición final de enunciado $((7)$ y $(11))$. Aparece también con frecuencia inserto en posición media (ejemplos (6), (8), (9), (13), (14), (15), (16), (18), (19)). Asimismo, puede también alcanzar tanto a predicados con verbo flexionado, como a otro tipo de predicados, como formas verbales no personales -participios (ejemplos (14) y (16))-, adjetivos (ejemplo (18)) o adverbios (ejemplo (7)).

En esos mismos trabajos, se ha puesto de manifiesto que, a partir de un significado originariamente temporal -la indicación de 'origen' o 'inicio' en el caso del operador en principio y la de 'actualidad temporal' en las locuciones por ahora y 
de momentolpor el momento-, estas expresiones desarrollan un significado modal epistémico de 'provisionalidad o contingencia' de la información a la que acompañan, como muestra el breve ejemplo siguiente y su correspondiente paráfrasis.

(5) En principio, Pepe vendrá.

['No aseguro que finalmente Pepe venga' / 'Tal vez Pepe (no) venga'].

En el siguiente ejemplo, procedente de corpus, la expresión en teoría muestra un significado epistémico semejante al de en principio consistente en debilitar la fuerza asertiva del miembro en el que aparece:

(6) -Y, bueno, qué ¿tienes realizas alguna actividad extraacadémica o algo de esto?

-Sí, al igual que mi compañero Pedro pertenezco a la asociación de estudiantes Programa Diez que es una asociación de allí, que está en Derecho, y que, bueno, tiene como objetivo la defensa de los intereses de los alumnos de esa facultad, y estamos allí trabajando duro y tal, ¿no? Y eso, esta actividad lo que te hace es perder mucho tiempo, ¿no?, porque podrías dedicarle más a los estudios y aparte de eso, ¿̨no?, mis padres siempre están diciéndome: chaval, deja esas actividades, ¿no?, y dedicate a lo que te va a dar de comer, ¿̇no?, que es ser abogado, [pero bueno, en teoría y eso] (...).

[CREA. Oral/ Grupo G 5: Derecho/ España/ 09.Formalidad=baja, Audiencia $=$ interlocutor, Canal $=$ cara a cara $]$.

Al insertar la partícula en teoría al final del enunciado, el hablante matiza la fuerza de la afirmación precedente "dedícate a lo que te va a dar de comer, que es ser abogado". De hecho, con la inclusión de este marcador, el hablante introduce la duda de que la carrera de Derecho le asegure realmente el sustento en el futuro.

Una función epistémica similar puede observarse en el ejemplo que sigue, en el que, para facilitar el análisis, hemos marcado con subrayado el fragmento que queda debilitado por la expresión en teoría:

(7) 374. A: $\quad$ // el Aese para los que si no conocéis

el Aese $\uparrow$ Apara que os hagáis una idea $\uparrow / / /$ el Aese es un sistema operativo dentro de un sistema operativo/ a ver si me explico/

(...) o sea el Aese e un mundo aparte $\downarrow$ e (...)

'(o sea que $)^{\circ} / / /$ es una cosa- es como el

Guorperfect que igual lo manejas en- bueno igual no/ pero igual lo manejas en Emesedós que en Güindous/ en teoría// pues esto es lo mismo ¿¿no? digamos que el Aese te independiza del sistema operativo [a un noventa y nueve por ciento]

(Briz y Val.Es.Co, 2002, conversación XP.48.A.1). 
$\mathrm{Al}$ igual que en el ejemplo previo de (6), en (7), al introducir la expresión en teoría (en este caso, al final de su enunciado a modo de restricción de la aserción precedente, siguiendo el esquema [x, en teoría]), el hablante debilita la fuerza asertiva del enunciado "Aese es como Guorperfect que igual lo manejas en Emesedós que en Güindous”. Con la inserción de esta partícula, el hablante permite que el interlocutor interprete la inferencia 'no aseguro que sea asi', 'tal vez no sea asi', 'en la práctica, eso no ocurre así. En teoría funciona, pues, como una señal de que el hablante no asume la verdad del enunciado que incluye el operador y, en consecuencia, no se compromete con él. Este valor epistémico del operador aparece recogido en el Diccionario de conectores y operadores (Fuentes 2009):

s.v. en teoría: "Limitativo de la aserción. Indica prevención del hablante ante lo dicho" (143). "Rebaja la fuerza asertiva y el compromiso con lo dicho" (144).

\section{EL SIGNIFICADO MODAL Y PRAGMÁTICO DE EN TEORÍA}

En los trabajos citados sobre operadores de debilitamiento de origen temporal, se indicó que, en el caso de dichos marcadores, la relación semántica de contraste temporal que acarrea el significado modal se establece entre, por un lado, lo que ocurre bien sea al inicio (principio) o bien en el momento actual (ahora/momento) que es, por definición, contingente $-y$, por tanto, informativamente débil-; y, por otro, lo que ocurre al final, que es lo definitivo y, por ello, lo relevante.

Por su parte, el valor debilitador de en teoría se basa, no en un contraste temporal, sino en el binomio dicotómico teoría-práctica ${ }^{5}$ de acuerdo con el cual existe una divergencia entre la teoría, que es especulativa, no está basada en hechos, no es de validez definitiva y resulta, por tanto, engañosa; y la práctica, que es empírica, real y, por consiguiente, fiable. La expresión en teoría introduce la posibilidad de que algo sea cierto sólo en un plano teórico; presupone, pues, una creencia compartida (una inferencia generalizada), basada en un contraste dicotómico que intenta reflejar la Tabla I:

\footnotetext{
${ }^{5}$ Para otras disociaciones filosófico-argumentativas que están en la base del significado de otras partículas discursivas de carácter argumentativo, véase el trabajo de Taranilla sobre los marcadores en realidad y realmente (2011).
} 
Tabla I. Tópico dicotómico en el que se basa la inferencia que acarrea en teoría.

\begin{tabular}{|c|c|}
\hline LA TEORÍA & LA PRÁCTICA \\
\hline $\begin{array}{c}\text { (no probada, no basada en hechos, no } \\
\text { definitiva) }\end{array}$ & (basada en hechos, definitiva, relevante) \\
Especulativa & Real \\
\hline
\end{tabular}

En la partícula en teoría no se ha borrado el significado de su componente léxico teoría, ya que el significado de este sustantivo tiene un valor relacional ${ }^{6}$ (en relación con la práctica), tal como se recoge en las entradas correspondientes de las principales obras lexicográficas del español ${ }^{7}$ (s.v. teoría):

DRAE: 1. f. Conocimiento especulativo considerado con independencia de toda aplicación. DEA ${ }^{8}$ : Conocimiento o razonamiento abstracto considerado con independencia de la realidad práctica.

DUE": 5. Por oposición a "práctica", conjunto de conocimientos sobre cierta actividad, separados de la práctica de ella.

De este significado de carácter relacional se entiende que el valor pragmático de en teoría se base en una oposición inferencial entre la teoría (especulación) y la práctica (realidad). Formulado en otros términos, la inserción de esta partícula discursiva dispara la implicatura generalizada de que es probable que no exista correspondencia entre teoría y práctica, tal como, de hecho, se recoge en los diccionarios:

DRAE: en teoría. Loc. adv. Sin haberlo comprobado en la práctica.

DUE: en teoría. Según la teoría, pero tal vez no confirmado en la práctica.

${ }^{6}$ En su análisis de la unidad en el fondo, que, en algunos de sus usos, también funciona como operador argumentativo, Delbecque considera el valor relacional del nombre fondo para explicar su estructura polisémica y su polifuncionalidad, pues fondo "tiene un significado espacial partitivo que remite a la representación esquemática de un espacio de referencia mayor del que es un componente constitutivo" (2012:248). Como se ve, también nosotros defendemos un valor relacional para el significado del sustantivo teoría que permite entender el valor pragmático de la partícula discursiva en teoría en términos de evocación inferencial de una alternativa contrastiva.

7 También recogen esta oposición en la definición de la unidad en teoría otro tipo de tratados lexicográficos, como el de Martínez y Jorgensen (2009): “Según la teoría pero sin ser confirmado por la práctica" (s.v. en teoría).

${ }^{8}$ Seco et al. (1999).

${ }^{9}$ Moliner (1984). 
La Figura 1 permite entender por qué el uso de en teoría evoca de inmediato otro plano, otro punto de vista, otra alternativa, el de la práctica o realidad, que se presenta, sea explícita o sea implícitamente, como el que probablemente sea verdadero, exacto y comprobado; el plano que verdaderamente sostiene el hablante.

Así, como muestran tanto los ejemplos presentados hasta aquí como los que siguen, el operador en teoría conlleva dos instrucciones inferenciales simultáneas. De un lado, el no compromiso del hablante respecto de la verdad o exactitud de lo dicho, inferencia de carácter epistémico derivada probablemente del hecho de que, como ya se ha señalado, el uso de en teoría apunta a otra información siempre comunicada aunque no siempre formulada: la información que contrasta con la marcada con el operador, la que corresponde al plano, no de la evanescente y poco fiable teoría, sino al plano de la realidad, como refleja la Figura 1:

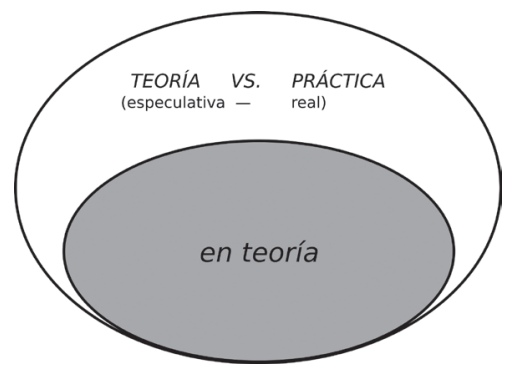

Figura 1.

Cabe un acercamiento a la interpretación de esta partícula según el modelo de la gramática cognitiva, del que se propone a continuación una sencilla aproximación por si puede ayudar a mostrar de un modo más claro su valor. Desde esta perspectiva teórica, como es sabido, la estructura semántica se entiende como una estructura conceptual convencionalizada, cuyo contenido es asimétrico en la medida en que está implicada una preeminencia cognitiva relativa, ya que, según Langacker $(1987,1991)$, una expresión adquiere su significado imponiendo su perfil a una base. La base puede definirse como la matriz subyacente de dominios cognitivos relevantes que se requiere o se evoca para comprender una expresión determinada, mientras que el perfil, a su vez, es la subestructura destacada sobre la base que la expresión en cuestión designa conceptualmente (Cuenca y Hilferty, 1999: 3.3.).

De acuerdo con ello, cabe proponer que la estructura semántica de en teoría tiene como base el binomio teoría-práctica, base sobre la que esta partícula destaca o perfila uno de los dos elementos del binomio y evoca inmediatamente el otro, como refleja la Figura 2, que utiliza los esquemas de representación del modelo de la gramática cognitiva (adaptado de Langacker, 2000): 
(a) la base

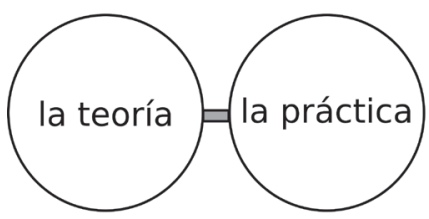

(b) en teoría

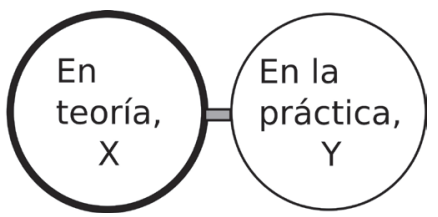

Figura 2.

El análisis del significado de este operador desde esta perspectiva teórica muestra de modo claro que el significado de en teoría no reside sólo sobre su base o sobre su perfil sino en la relación íntima entre la primera y el segundo; y en este sentido corrobora que "hay que darse cuenta de que la base es tan constitutiva de la estructura semántica como el perfil que aquélla contextualiza y por ello es igualmente importante analizarla" (Cuenca y Hilferty, 1999: 79).

En este caso, la enunciación de un determinado elemento lingüístico activa un esquema de conocimiento: lo que se marca como 'teórico' (en teoría) se presenta como no fiable, se refuta; e indirectamente se comunica que la correspondiente 'realidad' constituye la información fidedigna. Así, por ejemplo, en el anterior ejemplo de (7), la inserción de en teoría indica que el hablante se distancia de la afirmación "Guorperfect igual lo manejas en Emesedós que en Güindous”, y dispara la inferencia "en la práctica podría no ser asi”. Adviértase que, en algunos casos, incluso podría interpretarse "en realidad len el fondo, no A".

La Tabla II esquematiza la doble información, la dicha y la comunicada, que expresa esta partícula:

\section{Tabla II.}

\begin{tabular}{|c|c|}
\hline en teoría, A & $\begin{array}{c}\text { en la práctica, podría ser no A } \\
\text { (en realidadlde hecholen el fondo, } \mathbf{B})\end{array}$ \\
\hline LO DICHO & $\begin{array}{c}\text { LO COMUNICADO } \\
\text { (interpretación inferencial) }\end{array}$ \\
\hline INFORMACIÓN DEBILITADA & INFORMACIÓN PRIVILEGIADA \\
\hline
\end{tabular}

Por otro lado, nótese que es posible insertar cómodamente en el área sombreada, correspondiente a la información comunicada de forma inferencial y contrapuesta a la que señala en teoría, operadores de refuerzo argumentativo como en realidad, de hecho o en el fondo, de valor pragmático complementario al de los operadores de debilitamiento. Ello refuerza nuestra hipótesis del carácter de operador de debilitamiento argumentativo de esta partícula. 


\section{MÁS SOBRE LA OPOSICIÓN [EN TEORÍA - EN LA PRÁCTICA]}

Hasta el momento se han visto casos en los que la oposición teoría-práctica (o teoría-realidad) constituía un contraste de tipo inferencial. Por su parte, el ejemplo siguiente muestra un caso de oposición entre dos informaciones, la 'teórica' y la 'real', marcada de manera explícita, oposición que se presenta bajo un esquema sintáctico muy frecuente [en teoría $\mathrm{A}$, pero $\mathrm{B}]$ :

(8) si a lo mejor lo conoces/ o: no sé// bueno pues entonces que: // ella//

en teoría tiene/ una semana por la mañana/y otra por la tarde// pero

claro// como: es la jefe/// o la jefa/// entonces pues// (e:) hace

su turno// pero luego/ tiene que ir/ cuando no tiene que ir a pagar

lo de los- (e:) los seguros sociales// tiene que: el IVA cuando no tiene que:/ escribir a no sé quien/ cuando no (?) citado con un proveedor// y: bueno/ entonces/ (...)

(Moreno et al, 2002: 356).

Un aspecto de interés lo constituye el hecho de que, con frecuencia, este valor opositivo entre la teoría ('especulativa' y, por tanto, argumentativamente 'débil') y la práctica ('real' y, por consiguiente, 'fuerte' desde el punto de vista argumentativo) aparece explicitado por el mismo hablante, como muestran los ejemplos que siguen (9) y (10), en los que, a fin de facilitar el análisis, subrayamos los dos enunciados que se contraponen:

(9) Y luego la izquierda, pues sí, es la izquierda, es la o sea, la política en teoría la mejor que hay, porque es tú vive tu vida, no chafes al contrario, y eso, ¿̨no?, y repartir los bienes. Pero claro, que luego eso se lleve a la práctica, eso es muy distinto. Es que así no se progresa, la el socialismo puro pues todo el mundo sabe que no ha existido, y que no existirá, porque, claro, lo que ha dicho Nuria, vale, lo que tú has dicho, que tu padre es de derechas y tu madre no sé qué.

[CREA. Oral /España/ Grupo G11, Geografía e Historia /09.Formalidad=baja, Audiencia $=$ interlocutor, Canal=cara a cara $]$.

(10) Oficina de Información Turística, Del Salvador 328, Tel.: (65) 232.437, en teoría abre lun.-dom. de 09.00-21.00, en la práctica, casi siempre permanece cerrada, es preferible recurrir a Sernatur de Puerto Montt.

(CREA, Chile, G. Lux, Descubramos Chile, 1997).

El ejemplo siguiente muestra un caso interesante: un hablante replica a la intervención previa de su interlocutor, quien ha debilitado un enunciado mediante el uso de en teoría, y lleva a cabo dicha réplica a través de la apelación a la contraparte en (la) práctica: 
1437. E: [pero fíjate preguntan por ejemplo $\rightarrow$ ]/

(...) unas preguntas $\uparrow / /$

que en mi vida las había oído/ y yo voy to(do)s los días a la

autoescuela // supercomplicadas/ y preguntas que- bueno vamos

a ver $\rightarrow$ usted se encuentra ante un acccidentel ¿qué es lo primero que

hace? I/ mirar los heridos $\uparrow$ a ver qué tal están/ quitar el contacto o

señalizar el accidente $\downarrow$ bueno hay que hacer las tres cosas $\downarrow ¿ c u a ́ l ~ h a c e m o s$

antes $\uparrow$ ? pues no sé§

G: $\quad$ @la primera $\downarrow$ quitar el contacto

E: $\quad$ la primera $\downarrow$ quitar el contacto en teoría//[o sea pues]

L: $\quad$ [pues/ no lo entiendo]

G:

[no no no no no o

$s(\mathrm{e}) a]$ en teoría y en práctica... o sea§

(Briz y Valesco 2002. Conversación [L.15.A.2]).

\section{EN TEORÍA: DE SINTAGMA PREPOSICIONAL A PARTÍCULA}

En los últimos ejemplos propuestos ((9), (10) y (11)), la expresión en teoría muestra ya claros rasgos de pragmaticalización como modificador oracional y partícula, mientras que su contraparte, el sintagma en la práctica ${ }^{10}$, mantiene más características de complemento verbal y oracional. En el corpus manejado, se puede encontrar también casos en los que en teoría (equivalente a en la teoría) no funciona propiamente como partícula discursiva sino más bien como complemento verbal, como ocurre en el fragmento que aparece a continuación:

(12) - Ahora llega otro coche y se pone a su altura.

- Es él.

- Ella se está subiendo al otro coche.

- Perfecto. Eso es que van a alguna parte juntos. A ver si tenemos suerte y han quedado para algo más que besuquearse como adolescentes en un bar.

- Esto es horrible, Patricia. Tienes un trabajo odioso.

${ }^{10}$ Adviértase, no obstante, que en los ejemplos de (9), (10) y (11), la expresión en (la) práctica, que aparece como segundo miembro de la correlación "en teoríalen (la) práctica" presenta varias de las restricciones que se han señalado para en teoría, salvo la de la pérdida de determinante (pero atención al ejemplo (11)) y la de modificación. Es decir, la expresión en la práctica cancela parcialmente su sintaxis: (i) no tiene flexión de número: *en las/unas prácticas; (ii) aparece separada por pausa, al menos en posición marginal; (iii) tiende también a ocupar posiciones marginales. Ello parece apuntar a que, en estos ejemplos de correlaciones, en teoría presenta rasgos (la cancelación sintáctica) de una gramaticalización avanzada respecto de su homólogo en (la) práctica. (Agradezco esta observación a Fernando Polanco). 
- Pero Sofía, yo ya te expliqué de qué iba esto.

- Sí, pero no es lo mismo saberlo en teoría que comprobarlo en la práctica.

(CREA, L. Beccaria, La luna en Jorge, 2001).

Nótese que en la conversión desde sintagma preposicional hasta partícula discursiva, en teoría modifica forma y significado. Respecto de este último, como se ha visto, si bien mantiene su significado conceptual, adquiere la capacidad de disparar la inferencia convencional de la que se ha tratado en apartados anteriores. En relación con la forma, en teoría restringe sus posibilidades gramaticales y especializa sus características entonativas y de ubicación sintáctica. En concreto:

(i) bloquea su capacidad morfológica de flexión de número (compárese: en teoría/*en teorías, firmaremos mañana el contrato);

(ii) pierde el determinante (en teorial*en la teoríal ${ }^{*}$ en una teoría, firmaremos mañana el contrato);

(iii) bloquea la capacidad sintáctica de recibir modificadores ( ${ }^{*}$ en la teoría legal, firmaremos mañana el contrato);

(iv) aparece separado por pausas del resto del enunciado; y

(v) tiende a ocupar posiciones marginales del enunciado.

\section{SOBRE EL VALOR POLIFÓNICO}

Un aspecto de interés, ya que caracteriza especialmente a en teoría frente al resto de operadores de debilitamiento estudiados, lo constituye el hecho de que esta partícula presenta valor polifónico (Ducrot, 1982, 1984), en el sentido de que, como se ha visto, introduce de manera inferencial un punto de vista diferente al expresado por el enunciado señalado por en teoría del que el hablante se distancia críticamente, como indica Fuentes: "Apunta a otros enunciadores, de los que el hablante se desmarca” (2009: 144).

Es decir, la partícula en teoría conlleva una suposición alternativa ${ }^{11}$, suposición que, según los casos, puede corresponder a: (i) una formulación previa de otro locutor; (b) conocimiento compartido o (iii) una alternativa convocada por el propio hablante. Este valor polifónico de la partícula, que menciona un punto de vista

${ }^{11}$ En este sentido, recogiendo la propuesta de Portolés (2010a y 2010b, 2011), en teoría constituye un nuevo ejemplo de marcador del discurso que convoca al menos dos estados mentales intencionales y, en consecuencia, dos posibles puntos de vista, lo que, además de suponer una estrategia de distanciamiento asertivo del hablante, permite enriquecer el discurso con poco esfuerzo cognitivo. Como señala este autor, ambas voces o puntos de vista pueden atribuirse a un mismo locutor o a más de uno. 
distinto, explica que expresiones como sobre el papel ${ }^{12} \mathrm{o}$ en la letra, que claramente aluden a un enunciado establecido previamente, presenten un valor muy semejante de mitigación del compromiso asertivo. La Tabla III siguiente recoge este contenido polifónico del operador, que se suma al informativo y argumentativo:

\section{Tabla III.}

\begin{tabular}{|l|l|l|}
\cline { 2 - 3 } \multicolumn{1}{l|}{} & en teoría, A & en la práctica, B \\
\hline $\begin{array}{l}\text { NIVEL } \\
\text { ARGUMENTATIVO }\end{array}$ & $\begin{array}{l}\text { El hablante se distancia de A } \\
\text { (El hablante discute A) }\end{array}$ & $\begin{array}{l}\text { El hablante sostiene B } \\
\text { El hablante discute A para } \\
\text { defender explícita o implícita- } \\
\text { mente B }\end{array}$ \\
\hline $\begin{array}{l}\text { NIVEL } \\
\text { INFORMATIVO }\end{array}$ & Información debilitada & $\begin{array}{l}\text { Información reforzada } \\
\text { (a menudo domina la prosecu- } \\
\text { ción del discurso) }\end{array}$ \\
\hline $\begin{array}{l}\text { NIVEL } \\
\text { POLIFÓNICO }\end{array}$ & $\begin{array}{l}\text { Creación de un punto de } \\
\text { vista, A, con el que el locu- } \\
\text { tor no se compromete. } \\
\text { A puede haber sido dicho } \\
\text { por otro hablante }\end{array}$ & $\begin{array}{l}\text { El hablante comunica B } \\
\text { (lo diga o no de manera ex- } \\
\text { plícita) }\end{array}$ \\
\hline
\end{tabular}

\section{EN TEORÍA, OPERADORES DE DEBILITAMIENTO Y PATRONES DISCURSIVOS}

En apartados anteriores se ha mostrado que, probablemente porque su significado está basado en un tópico que contrasta dos conceptos, el uso de en teoría apela siempre a la existencia de un estado de cosas alternativo al señalado por el marcador. La apelación a esa alternativa, como se ha visto, en ocasiones constituye una estrategia de salvaguarda de la imagen del propio hablante, debilitando su aserción al implicitar una posibilidad diferente a la enunciada (en teoría, $\mathrm{A}$ = podría no ser A). En otras ocasiones, en cambio, la alternativa convocada por en teoría constituye el estado de cosas real que es el que, en el fondo, sostiene el hablante. Por

${ }^{12}$ Como muestra el siguiente ejemplo, en el que la partícula sobre el papel debilita el enunciado al que afecta y, al mismo tiempo, anuncia un movimiento posterior en el discurso de tipo contraargumentativo (que se ha marcado en negrita). De hecho, Santos ya advierte el valor anticipativo de contraargumentación que suele expresar esta partícula (2003: 486).

"Salió Andre Agassi a la pista central de Flushing Meadows con la sonrisa amplia de los campeones. Estaba convencido que su estrella iba a brillar con autoridad en la noche iluminada de Nueva York. El rival, sobre el papel, parecía un buen "sparring" para darse un paseo en tres sets camino de la renovación de su título. (...) Pero no. Alex Corretja no se arrugó. Le tomó el pulso al ambiente. Apretó los dientes. Tensó sus músculos. (...) y amenazó con dejar sin cabeza el Open de Estados Unidos. Le faltó muy poco. Un set nada más". (CREA, La Vanguardia, 02/09/1995). 
ello, no sorprende que, al igual que ocurría con los operadores de debilitamiento argumentativo de origen temporal, en teoría funcione de manera recurrente como marcador predictivo de un movimiento contraargumentivo de tipo adversativo en miembros siguientes ${ }^{13}$.

Así, los ejemplos anteriores (8) y (9) reflejan que, con frecuencia, el inicio del movimiento contraargumentativo posterior a la inserción de en teoría se marca mediante la conjunción pero, que introduce un miembro de carácter adversativo, adyacente al miembro debilitado previamente por la presencia de esta partícula, respondiendo a la construcción o patrón discursivo [en teoría, A. Pero B $]^{14}$.

Los ejemplos siguientes, en cambio, muestran cómo en ocasiones ese movimiento de contraargumentación puede aparecer a mayor distancia del miembro discursivo debilitado. Así ocurre en los casos de (13) y (14), en los que el miembro adversativo encabezado por pero no está contiguo al debilitado por en teoría sino alejado sintácticamente, con la inserción de una oración subordinada adverbial impropia condicional (ocho palabras) en el ejemplo (13) o de varias oraciones complejas (62 palabras) en el ejemplo de (14):

(13) Los pedalieres sellados de Shimano en teoría no se pueden desmontar. Si no te da problemas, ni lo intentes; pero si se te queda agarrotado, hay recursos antes de tirarlo. El primero es intentar quitar los guardapolvos (las gomas, vamos) que cubren los rodamientos. Esto nos permite acceder a los mismos, para limpiarlos mejor y engrasarlos. En último extremo, se puede desmontar el eje.

(CREA, Efímero, 99206009. Página web 1999).

(14) Afortunadamente tampoco ha habido grandes problemas en la Vuelta [ciclista]. No, afortunadamente. Bueno, por lo menos que hayamos visto nosotros desde aquí, ¿̨no? Pero el hecho es *de que*, bueno, que es justo que le le abronque [el Director] al corredor porque como eso está, en teoría, prohibido, pues tiene que abroncar, porque si no a él le dicen: “*Oyes*, ¿̇ú qué pintas ahí?” el juez principal ¿̨no?, o sea, *ro lógico que* él tenga que hacer valer un poco por qué está ahí y *hacer valer un poco la carrera cómo tiene que desarrollarse*. Sabe que es una circunstancia de carrera y que se puede decir que es sancionable, perono hasta el punto de decirle que no puede... o sea, quitarle incluso la victoria, sabe que eso sería ilógico, pero sí, bueno, pues darle el toque de atención de que eso no es así, y y es bueno, ¿̇no? Yo también estoy un poco de acuerdo con lo que dice Pello.

[CREA. Oral/ Vuelta ciclista a España, 24/09/95, TVE 1/ España/ 09.

Retransmisiones deportivas].

\footnotetext{
${ }^{13}$ Tal y como señala Santos acertadamente en su Diccionario de partículas en la entrada correspondiente a en teoría: "A menudo anticipa una restricción adversativa fuerte" (2003: 625).

${ }^{14}$ Para otros trabajos que abordan también la potencialidad del estudio de los marcadores y de los patrones discursivos desde una perspectiva construccionista, puede verse Polanco (en prensa).
} 
Dada la posibilidad de planificación consustancial a los textos escritos, los ejemplos de este registro muestran que el movimiento contraargumentativo puede aparecer todavía más alejado, a saber: en el párrafo siguiente. De hecho, cabe, pues, interpretar la utilización de en teoría en algunas ocasiones como un mecanismo de conexión discontinua entre párrafos a través de una doble marcación (en teoría-Sin embargo), como refleja el ejemplo de (15):

(15) Según acabamos de exponer, la temperatura ambiental conseguida por la calefacción, en teoría no debería ser uniforme para toda la vivienda, sino que variará de acuerdo con las circunstancias determinantes de cada habitación. También debe tenerse en cuenta la actividad que se desarrollará, normalmente, en las distintas piezas. Una habitación destinada a pequeño taller, necesitará menos temperatura que otra dedicada a sala de lectura, de la misma manera que una cocina o el cuarto de planchar serán mucho más soportables a 17 ${ }^{\circ} \mathrm{C}$ que, por ejemplo, el recibidor.

Sin embargo, en la mayoría de las instalaciones de calefacción solar, tal regulación no es posible, ya que el circuito de transmisión calórica está constituido por tuberías conductoras empotradas en el suelo. Por ello, debe buscarse la temperatura media más apropiada para toda la casa, que para unos pueden ser los $20^{\circ} \mathrm{C}$ y para otros los $22^{\circ} \mathrm{C}(\ldots)$.

(CREA: J. de Cusa, Energía solar para viviendas. 1998).

Las ocurrencias de lengua escrita muestran también que la marcación antiorientada del segundo miembro respecto del previo puede realizarse a través de otros medios diferentes a la conjunción pero, como, por ejemplo, otros conectores adversativos como no obstante, con todo o sin embargo (ejemplo (15)); o mediante operadores de refuerzo argumentativo como de hecho o en realidad (ejemplo (16)); o bien a través de procedimientos léxicos con cierto valor conectivo, como lo cierto es que o la realidad es que (ejemplo (17)):

(16) Gran parte de las publicaciones dedicadas en teoría a la cobertura de la información sobre el espectáculo, no son de hecho sino extensiones del mismo: música y tendencias, entre otros, son contenidos que dan lugar a publicaciones especializadas en las que lo de menos es el rigor en la valoración crítica, y lo de más la creación y explotación de mitos.

(CREA. P. Baeza, Por una función crítica de la fotografía de prensa. 2001).

(17) Pensé en la cura del opio de Bill Burroughs. Se trataba de introducir en una botella una solución de agua y opio. Cada vez que te inyectabas un "chute" de la botella, debías devolver con la jeringuilla la misma cantidad de agua pura al recipiente. Al final, en teoría, deberías acabar inyectándote solo agua cristalina. Lo cierto es que todos los yonquis terminaban haciéndose trampas a sí mismos y nunca conocías a nadie a quien le hubiera funcionado el asunto de la botella.

(CREA. S. Méndez, Corre, rocker. Crónica personal de los ochenta. 2000). 
Los ejemplos utilizados hasta aquí nos permiten observar que en teoría, como los operadores de debilitamiento, en general, forma parte de un patrón discursivo de contraargumentación anticipada, como puede comprobarse cuando se sistematizan los ejemplos analizados:

\section{Tabla IV.}

(9) La izquierda es en teoría la política mejor que hay

(10) En teoría, abre de lunes a domingo de 9 a 21 hs.

(13) Los pedalieres, en teoría, no se pueden desmontar

(14) Eso en teoría está prohibido

(15) La temperatura en teoría no debería ser uniforme en toda la vivienda

(16) Al final, en teoría, deberías acabar inyectándote solo agua cristalina pero claro que todo eso se lleve a la práctica es muy distinto

(pero) en la práctica casi siempre permanece cerrada

pero en último extremo se puede desmontar el eje.

Pero no hasta el punto de quitarle incluso la victoria al corredor

Sin embargo, generalmente la regulación no es posible.

Lo cierto es que nunca conocías a nadie a quien le hubiera funcionado el asunto de la botella

LA PRÁCTICA, LOS HECHOS aserción reforzada

Refutación del miembro anteriormente debilitado

\section{PATRÓN DISCURSIVO DE TIPO ARGUMENTATIVO}

En un primer movimiento argumentativo, el hablante inserta el operador de debilitamiento en teoría, que, como se ha señalado, por un lado, marca el constituyente o enunciado al que señala como debilitado o discutido y, por otro, anuncia un segundo miembro posterior que estará argumentativamente antiorientado y que refutará el enunciado previo. El segundo miembro propone la versión que, en el fondo, defiende el hablante, versión que constituye una refutación ${ }^{15}$ respecto de la afirmación previamente debilitada ${ }^{16}$. Como muestran los ejemplos de la figura anterior, este segundo miembro con frecuencia aparece marcado, a su vez, con un marcador de tipo adversativo o contrastivo.

${ }^{15}$ La estrecha relación que existe entre el uso de en teoría y la refutación se refleja en la frecuencia con la que aparecen estructuras sintácticas de tipo refutativo [no A sino (que) B] en los ejemplos analizados, como muestra el caso de (14).

${ }^{16}$ Un valor semejante a en teoría tiene el adverbio teóricamente. Véase la nota 10 para las semejanzas con la locución sobre el papel. 


\section{Tabla V.}

PATRÓN DISCURSIVO DE CONTRAARGUMENTACIÓN ANTICIPADA

\begin{tabular}{|l|l|}
\hline \multicolumn{1}{|c|}{$1^{\text {er }}$ miembro } & \multicolumn{1}{c|}{$2^{\circ}$ miembro } \\
\hline operador de debilitamiento, A & $\begin{array}{l}\text { (marca adversativa o de refuerzo } \\
\text { argumentativo), B }\end{array}$ \\
\hline $\begin{array}{l}\text { (5) en teoria la izquierda es la mejor poli- } \\
\text { tica que hay }\end{array}$ & $\begin{array}{l}\text { pero } \text { claro, que luego eso se lleve a la prácti- } \\
\text { ca, eso es muy distinto }\end{array}$ \\
\hline $\begin{array}{l}\text { MIEMBRO PREDICTIVO } \\
\text { ARGUMENTATIVAMENTE DEBILITADO } \\
\text { (ELEMENTO DISCUTIDO) }\end{array}$ & $\begin{array}{l}\text { MIEMBRO ANTIORIENTADO } \\
\text { ARGUMENTATIVAMENTE FUERTE. } \\
\text { REFUTACIÓN DEL MIEMBRO } \\
\text { ANTERIOR }\end{array}$ \\
\hline
\end{tabular}

Este patrón discursivo es el resultado lingüístico de un proceso de rutinización de estrategias argumentativas de antiorientación anticipada. Que los hablantes reconocen el contraste adversativo que acarrea siempre en teoría entre, por un lado, la "teoría” mencionada para discutirla, y la "práctica", los "hechos", o la "verdad", que constituyen el enunciado que verdaderamente sostiene el hablante se comprueba en ejemplos como (11), en el que, como se ha visto, el hablante refuta la discusión que su interlocutor había iniciado al utilizar en teoría en su enunciado, siguiendo el esquema: - "X en teoría" / --"No no no no. En teoría y en la práctica”.

En menos ocasiones, la debilidad asertiva y argumentativa del enunciado que contiene en teoría se refuerza mediante la utilización de marcas conjuntivas de tipo concesivo; en tales casos, dado que se marca doblemente la debilidad argumentativa del primer miembro, no es preciso utilizar marca de adversación en el segundo:

(18) Yo les explicaba lo mejor que podía que aquello era como una vacuna, o sea, la inoculación del virus auténtico en dosis tolerables, para estar inmunizados contra las nuevas formas totalitarias; pero [ REForzado siempre sospeché que no se fiaban del todo]. Tenían su parte de razón, porque por encima de cualquier altruismo, me invadía el gusto de jugar perversamente con el público.

(CREA. A. Boadella, Memorias de un bufón. 2001).

Este tipo de ejemplos muestra la participación del operador en teoría en estrategias argumentativas de construcción colegiada de la debilidad argumentativa de un segmento textual. Así, en el fragmento siguiente de (19), puede observarse la coparticipación de diferentes mecanismos gramaticales para acentuar la insufi- 
ciencia argumentativa del enunciado ${ }^{17}$ "separar la pequeña y mediana minería de la gran minería”; a saber: (i) la inclusión del enunciado en una cláusula concesiva mediante la inserción de aunque al inicio; (ii) la flexión del verbo modal querer en imperfecto de subjuntivo; y (iii) la presencia del operador de debilitamiento en teoría:

(19) Otra arista de este asunto la constituye la situación de la pequeña y mediana minerías,

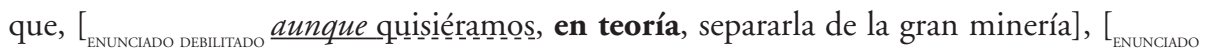
resultaría imposible hacerlo]. Lo que ocurra en el conjunto del negocio a nivel mundial repercutirá de manera directa en ese sector del quehacer nacional, que comprende una vasta zona del país: desde parte de la Segunda Región hasta la Quinta Región, comprometiendo a pueblos enteros y a miles de compatriotas que no tienen posibilidades de llevar a cabo otra actividad. Éste no es sólo un problema económico, sino también social, e incluso cultural.

(CREA. Oral. Chile. Sesión 9, en martes 9 de noviembre de 1999.formalidad=alta, audiencia $=$ interlocutor, canal=cara a cara).

Como puede verse, los anteriores ejemplos (18) y (19) ilustran un patrón discursivo de argumentación algo diferente [conector concesivo + en teoría, A /B], alternativo al patrón [en teoría, A / Conector adversativo, B] que se ha expuesto en los apartados precedentes.

\section{CONCLUSIONES}

El análisis desarrollado a lo largo de los diferentes apartados muestra que la locución adverbial en teoría funciona en determinados contextos sintácticos como una partícula discursiva con valor modal epistémico, partícula que el hablante utiliza para debilitar la fuerza asertiva del enunciado $y$, en consecuencia, para distanciarse estratégicamente de su compromiso con la exactitud de éste.

Tal valor pragmático del marcador en teoría procede del significado léxico del sustantivo teoría, de carácter relacional, ya que teoría se define en una de sus acepciones como lo opuesto a la práctica. Por ello, en línea con el significado procedimental de otros marcadores del discurso, en tanto que partícula, en teoría convoca

${ }^{17}$ En trabajos previos dedicados al estudio de los operadores de debilitamiento de origen temporal, se ha analizado este mismo fenómeno de construcción de la debilidad argumentativa de un miembro del discurso a través del uso simultáneo de diferentes procedimientos gramaticales, entre los que destaca la inserción de uno de estos operadores (Montolío, 2011). 
una alternativa, un escenario inferencial disyuntivo, el correspondiente a la esfera de la práctica o realidad, en la que proposición enunciada podría no cumplirse. De ahí, también, el valor polifónico de esta unidad, que se ha analizado en el apartado 7. Se han utilizado herramientas de la lingüística cognitiva, como los conceptos de perfil y base, para explicar el valor inferencial de esta unidad.

Se ha mostrado también que, en determinados usos, en teoría funciona como un operador argumentativo, ya que cumple con las características utilizadas por Ducrot para definir estos elementos. Más concretamente, se propuso que en teoría funciona en tales casos como un operador de debilitamiento argumentativo, en una línea semejante a la de operadores argumentativos de este mismo tipo, como en principio o por ahora, puesto que, al igual que ocurre con estos últimos operadores, la inserción de en teoría en un enunciado determina la orientación argumentativa en la prosecución del discurso.

En el apartado 8 se sostuvo que este marcador suele formar parte de un patrón discursivo, que se encuentra tanto en registros orales como escritos, patrón en el que la partícula en teoría funciona como una marca predictiva de un movimiento de contraargumentación posterior que puede aparecer a cierta distancia en el discurso, y no necesariamente en la cláusula siguiente a la que contiene este marcador. Es decir, el uso de en teoría está ligado con abrumadora frecuencia a la aparición de un patrón discursivo de contraargumentación anticipada del tipo [en teoría, A / marca de contraargumentación (o de fuerza argumentativa), B], en el que la inserción de la partícula en teoría desempeña la doble función de debilitar la fuerza asertiva de una de las proposiciones en liza y de avisar del movimiento de refutación posterior.

Futuros trabajos deberían investigar y esclarecer en qué consisten exactamente las semejanzas y diferencias, en cuanto a su valor epistémico y de debilitamiento argumentativo, entre las diferentes partículas que, en diferentes artículos, se han analizado e incluido en el grupo de los operadores argumentativos de debilitamiento; a saber: en principio, por ahora, de momento, por el momento y en teoría.

Igualmente, se espera haber abierto una línea en el ámbito de la investigación sobre los marcadores del discurso que se ocupe no sólo de la conexión en contigüidad sintáctica sino también de la conexión textual que se establece entre miembros del discurso no contiguos, como se ha observado en la partícula discursiva anticipativa de contraargumentación en teoría. Por otro lado, se propone ahondar próximamente en el estudio del enfoque construccionista y de patrones discursivos que permita explicar el importante porcentaje de casos que muestra el corpus de ocurrencias de este marcador que responden al esquema [al menos/por lo menos en teoría] o [aunque en teoría]. 


\section{REFERENCIAS}

Anscombre, Jean-Claude y Ducrot, Oswald. (1983). L'argumentation dans la Langue. Brux-elles: Mardaga.

Briz, Antonio y Grupo Valesco. (2002). Corpus de conversaciones coloquiales. Madrid: Arco/Libros.

Briz, Antonio; Pons, Salvador y Portolés, José (coords.). (2008). Diccionario de partículas discursivas del español (DPDE). [En línea] http://www.dpde.es

Cuenca, Ma Josep. (1990). "Els matisadors: connectors oracionals i textuals", Caplletra, 8, 149-167.

Cuenca, Ma. Josep. (2001). Los conectores parentéticos como categoría gramatical, Lingüística Española Actual 23(2), 211-235.

Cuenca, Ma. Josep y Joseph Hilferty. (1999). Introducción a la lingüistica cognitiva. Barcelona: Ariel.

Delbecque, Nicole. (2012). En el fondo: polifuncionalidad y polifonía de la localización interna, en Jiménez Juliá, Tomás, López Meirama, Belén, Vázquez Rozas, Victoria, Veiga Rodríguez, Alexandre (Eds.) Cum corde et in nova grammatica. Estudios ofrecidos a Guillermo Rojo. Santiago de Compostela: Servizo de Publicacións e Intercambio Científico da Universidade de Santiago de Compostela, 247-263.

DRAE. (1992). Diccionario de la Lengua Española (21ª edición). Madrid: Espasa Calpe.

Ducrot, Oswald. (1982). La notion de sujet parlant. En Recherches sur la philosophie et le langage, vol. 2, 65-93.

Ducrot, Oswald. (1984). Esquisse d'une théorie polyphonique de l'énonciation. En Le dire et le dit. Paris: Minuit, 171-233.

Fuentes, Catalina. (1987). Enlaces extraoracionales. Sevilla: Alfar.

Fuentes, Catalina. (2009). Diccionario de conectores y operadores del español. Madrid: Arco/Libros.

Hyde, John. (1999). Lo demás es literatura. Predicción y adversatividad en textos argumentativos en inglés y español, en J. Fernández González et alii [Eds.], Lingüistica para el siglo XXI (vol II). Salamanca: Ediciones de la Universidad de Salamanca, 935-941.

Hyland, Ken. (2005). Metadiscourse. Exploring interaction in writing. LondresNueva York: Continuum.

Langacker, Ronald. (1987). Foundations of Cognitive Grammar vol I: Theoretical Prerequisites. Stanford: Standford University Press.

Langacker, Ronald. (1991). Foundations of Cognitive Grammar. volume II: Descriptive Application. Stanford: Standford University Press.

Langacker, Ronald. (2000). Concept, Image, and Symbol: The Cognitive Basis of Grammar. Berlin: Mouton de Gruyter. 
Martín Zorraquino, Ma Antonia y Portolés, José. (1999). Los marcadores del discurso, en Bosque, Ignacio y Demonte, Violeta (Dirs.). Gramática Descriptiva de la Lengua Española (vol. 3). Madrid: Espasa-Calpe.

Martínez, Juan Antonio y Myre Jorgensen, Annette. (2009). Diccionario de expresiones y locuciones del español. Madrid: Ediciones de la Torre.

Moliner, María. (1984). Diccionario de uso del español. Madrid: Gredos.

Montolío, Estrella. (2001). Conectores de la lengua escrita. Barcelona: Ariel.

Montolío, Estrella. (2003). Es una buena periodista, en principio. Sobre el operador discursivo en principio y su función modalizadora en el discurso periodístico, Español Actual 79, 45-58.

Montolío, Estrella. (2011). Mitigación del compromiso asertivo y mecanismos argumentativos en la oralidad: los operadores de debilitamiento, en Fant, Lars y Harvey, Ana María (Eds.). El diálogo oral en el mundo hispanohablante. Frankfurt am Main: Iberoamericana/Vervuet, 95-118.

Montolío, Estrella. (2014). 'Por ahoral de momentol por el momento, es un tipo encantador'. Operadores de debilitamiento argumentativo de origen temporal, en Falk, Johan; Gille, Johan y Wachtmeister, Fernando (Eds.). Discurso, interacción e identidad (2a edición). Estocolmo: Stockholms Universitet, 81-107.

Montolío, Estrella. (2016). Mecanismos de cohesión (II). Los conectores, en Montolío, Estrella (Dir.) Manual de escritura académica y profesional (2a edición, vol. II). Barcelona: Ariel, 11-92.

Moreno, Francisco; Ana Ma Cestero; Isabel Molina y Florentino Paredes. (2002). La lengua hablada en Alcalá de Henares. Corpus PRESEEA. Hablantes de instrucción media. Alcalá: Universidad de Alcalá.

Polanco Martínez, Fernando. (2013). Redes polisémicas y niveles de interpretación. Representación semántica de unidades lingüísticas complejas: el caso de vamos, Estudios de Lingüística de la Universidad de Alicante 27, 199-250.

Polanco Martínez, Fernando. (2014). Variación funcional y polisemia en la descripción de marcadores conversacionales en español peninsular: el caso de vaya. Revista de la Sociedad Española de Lingüistica, 44/2, 131-164.

Polanco Martínez, Fernando. (en prensa). Marcadores del discurso y contextos funcionales: la reformulación como construcción discursiva, Onomázein, 34.

Portolés, José. (1998). Marcadores del discurso, Barcelona: Ariel.

Portolés, José. (1999). Los marcadores del discurso. Barcelona: Ariel.

Portolés, José. (2004). Pragmática para hispanistas. Madrid: Síntesis.

Portolés, José. (2010a). Alternativas convocadas por partículas discursivas. Español Actual 92, 47-68.

Portolés, José. (2010b). Los marcadores y la estructura informativa, en Loureda Óscar y Esperanza Acín (coords.). La investigación sobre marcadores del discurso en español, hoy. Madrid: Arco/Libros, 281-325.

Portolés, José. (2011). Las partículas focales desde una perspectiva polifónica, en 
Aschenberg, Heidi (Ed.). Marcadores del discurso: de la descripción a la definición. Madrid/Frankfurt: Iberoamericana/Vervuet, 51-76.

Santos Río, Luis. (2003). Diccionario de partículas. Salamanca: Luso-española de ediciones.

Seco, Manuel; Andrés, Olimpia; Ramos, Gabino. (1999). Diccionario del español actual. Madrid: Aguilar.

Taranilla, Raquel. (2011). En realidad/ realmente, tú ya no me quieres. Partículas discursivas basadas en el valor argumentativo de lo real, en González Ruiz, Ramón (Ed.): Nuevos estudios sobre los marcadores del discurso. Pamplona: Eunsa.

Tadros, Angelika. (1994). Predictive Categories in Expository Texts, en Coulthard, Malcom (Ed.), Advances in Written Text Analysis. London - New York: Routledge: 69-82.

Yúfera, Irene. (2014). Exponer información. Estrategias para la construcción de textos expositivos. En Montolío Durán, Estrella (Dir.) Manual de escritura académica y profesional (vol. II). Barcelona: Ariel, 121-186. 